\title{
The first presentation of diabetes in a four-month-old infant with diabetic ketoacidosis (DKA) precipitating by COVID-19: a case
} report

\author{
Elham Maleki ${ }^{1}$, Amir Baniasad ${ }^{1}$, Mina Sepehran $^{2}$, and Najmeh Davoudian ${ }^{3}$ \\ ${ }^{1}$ Kerman University of Medical Sciences Faculty of Medicine \\ ${ }^{2}$ Kerman University of Medical Sciences \\ ${ }^{3}$ Gonabad University of Medical Sciences
}

August 18, 2021

\begin{abstract}
Diabetes mellitus is rare in infants and causes a high mortality when complicated with diabetic ketoacidosis (DKA).The concomitant of COVID-19 and DKA in four-month infant with first presentation of neonatal diabetes shows that COVID-19 can accelerate the onset of DKA, and we should consider this in the clinical context.

The first presentation of diabetes in a four-month-old infant with diabetic ketoacidosis (DKA) precipitating by COVID-19: a case report

Abstract

Diabetes mellitus is rare in infants and causes a high mortality when complicated with diabetic ketoacidosis (DKA).The concomitant of COVID-19 and DKA in four-month infant with first presentation of neonatal diabetes shows that COVID-19 can accelerate the onset of DKA, and we should consider this in the clinical context.
\end{abstract}

\section{Keywords}

Coronavirus disease 2019 (COVID-19), DKA (diabetic ketoacidosis), Hyperglycemia, neonatal diabetes

\section{Key Clinical Message}

DKA, as an early manifestation of diabetes at infancy, can be accelerated by COVID-19 infection, such that distinguished diagnosis becomes imperative.

\section{Introduction}

In late 2019, a new coronavirus was identified as the cause of pneumonia in China, which spreads rapidly and becomes a pandemic around the world (1). Coronavirus disease 2019 (COVID-19) can affect any age group and has different clinical manifestations. Pneumonia seems to be the most common and severe manifestation of COVID-19 infection. It is characterized by fever, coughs, and dyspnea but asymptomatic infections have also been reported $(2,3)$.

COVID-19 is caused by severe acute respiratory syndrome coronavirus 2 (SARS-CoV-2) and it enters the cells by binding to ACE2 receptors. These receptors are present in the body's major organs and tissues, including lung, pancreatic cells, adipose tissue, small intestine, and kidneys (4). The virus may lead to acute dysfunction of pancreatic beta cells, leading to acute hyperglycemia (5). 
This study aimed to report a four-month-old infant whose first manifestation of diabetes was diabetic ketoacidosis. Diagnosis of COVID-19 infection after admission is considered as a possible cause of accelerated diabetic ketoacidosis (DKA) in our patient.

\section{Case Presentation}

A four-month-old infant boy was presented to the pediatric emergency department of Afzalipour Hospital in Kerman, Iran with a two-day history of fever, tachypnea, and vomiting several times. The infant was the first child of the family and the parents were relative. The infant had respiratory distress, drowsiness, and severe dehydration at the time of admission. Upon admission, infant's anthropometric measures were weight $5.5 \mathrm{~kg}$, height $63 \mathrm{~cm}$ and the vital signs were temperature $39^{\circ} \mathrm{C}$, respiratory rate 52 per minute, heart rate 180 per minute, blood pressure of $75 / 50 \mathrm{mmHg}$, and oxygen saturation of $95 \%$ in room air.

The laboratory findings (as shown in Table 1) confirmed diabetic hyperglycemia and diabetic ketoacidosis. Chest computed tomography (CT) scan was reported unifocal ground-glass opacity in the apical segment of right upper lobe (RUL) (figure 1). Nasopharyngeal swab for reverse transcription-polymerase chain reaction (rRT-PCR) test was positive for SARS-COV-2. The results of blood and urine cultures were negative. Parents did not report contact with any suspected febrile patient.

Figure 1: Unifocal ground-glass opacity in the apical segment of right upper lobe (RUL) of the patient

Treatment with isotonic fluids and intravenous insulin was started for him and he was transferred to the pediatric intensive care unit (PICU). Regular monitoring of vital signs, electrolytes, and blood sugar was performed. Apart from supportive therapy, no other treatment was started for COVID-19. Gradually, his level of consciousness and general condition improved and after two days DKA was resolved so breastfeeding and subcutaneous neutral protamine Hagedorn $(\mathrm{NPH})$ insulin were started for the patient.

The starting dose of NPH insulin for the patient was 1.5 units at night. Blood glucose was measured regularly to adjust the insulin dose. The patient's insulin dose was gradually increased to one unit in the morning and one and a half units at night. After three days of admission his fever subsided and after 7 days of admission, he was discharged.

\section{Discussion}

In this study, we reported a four-month-old infant whose first manifestation of diabetes was DKA. Most cases of diabetes that present in the first six months of life have a monogenic cause and are known as neonatal diabetes (6). Diabetes is a rare cause of infantile and neonatal hyperglycemia. In some cases, DKA is the first manifestation of diabetes (7).

DKA is a metabolic disorder characterized by the following biochemical criteria: $\mathrm{PH}<7.3$, Serum HCO3 $<15$, and with serum glucose greater than $200 \mathrm{mg} / \mathrm{dl}$ and the presence of concomitant ketones in the blood $>3 \mathrm{mmol} /$ lit or urine ketones [?]2+ (8). Infections are common and known causes that accelerate the onset of DKA $(9,10)$.

Soliman et al. (11) reported a case of DKA in an eight-month-old infant infected by COVID-19. In another study, Suwanwongse et al. (12) reported three cases of COVID-19 newly diagnosed diabetes mellitus. In the first case, a concurrent between COVID-19 and diabetes was observed, and in the other two cases, the patient was diagnosed with diabetes after COVID-19 infection. Although, the cases reported in their study were adults.

The mechanism by which COVID-19 induces DKA is not known, but direct damage to pancreatic beta cells due to infection and acute insulin depletion can lead to DKA (13). Localization of ACE2 in the endocrine region of the pancreatic cells suggests that SARS-CoV-2 through these receptors enters the cells of the pancreas and causes cell damage (14).

There are few reports of the early manifestation of type 1 diabetes and its association with infection by COVID-19 $(11,15)$. Our knowledge of COVID-19 and diabetes is still limited. The infant was diagnosed 
with neonatal diabetes due to age and negative antibodies, the first manifestation of which was diabetic ketoacidosis and concomitant with COVID-19. Despite the severity of ketoacidosis and its association with COVID-19, the patient responded well to the treatment and was discharged with the order of subcutaneous insulin at home.

\section{Conclusion}

The combination of COVID-19 and DKA, in this case, shows that COVID-19 can accelerate the onset of DKA and we should consider it in the clinical context. DKA, as an early manifestation of diabetes at infancy, can be precipitated due to COVID-19 infection. Concurrence of COVID-19 and DKA can have devastating effects if proper treatment is not started immediately.

\section{Author contributions}

EL and MS: the endocrinologist involved in treating the patient and collaboration in writing and editing the case report.

MS: the other endocrinologist involved in treating the patient and collaboration in editing and submitting the manuscript.

$\mathrm{AB}$ and ND: Authors of the initial draft of the manuscript and editing the article.

\section{Conflict of interest}

There is no conflict of interest.

\section{Funding}

There is no external funding source for this case report.

\section{Ethical approval}

The informed consent of the patient's parents has been obtained and the therapy procedure has been approved by the "Iran National Committee for Ethics in Biomedical Research" (http://ethics.research.ac.ir/IndexEn.php, no.: IR.KMU.AH.REC.1400.025).

\section{Data availability}

The data that support the findings of this study are available from the corresponding author upon reasonable request.

\section{References}

1. Poursadegh F, Davoudian N, Mozdourian M, Abdollahi F. Synchronous presentation of COVID-19 pneumonia and pulmonary embolism. Clinical case reports. 2021;9(3):1663-6.

2. Ekrami Noghabi M, Baniasad A, Heidari E, Davoudian N, Malekzadeh F. A 35-Day Old Infant with COVID-19. Iran J Pediatr. 2020;30(4):e103807.

3. Docherty AB, Harrison EM, Green CA, Hardwick HE, Pius R, Norman L, et al. Features of 20133 UK patients in hospital with covid-19 using the ISARIC WHO Clinical Characterisation Protocol: prospective observational cohort study. BMJ (Clinical research ed). 2020; 369:m1985.

4. Hamming I, Timens W, Bulthuis ML, Lely AT, Navis G, van Goor H. Tissue distribution of ACE2 protein, the functional receptor for SARS coronavirus. A first step in understanding SARS pathogenesis. The Journal of pathology. 2004;203(2):631-7.

5. Hoffmann M, Kleine-Weber H, Schroeder S, Kruger N, Herrler T, Erichsen S, et al. SARS-CoV-2 Cell Entry Depends on ACE2 and TMPRSS2 and Is Blocked by a Clinically Proven Protease Inhibitor. Cell. 2020;181(2):271-80.e8. 
6. Rubio-Cabezas O, Ellard S. Diabetes mellitus in neonates and infants: genetic heterogeneity, clinical approach to diagnosis, and therapeutic options. Hormone research in paediatrics. 2013;80(3):137-46.

7. Letourneau LR, Carmody D, Wroblewski K, Denson AM, Sanyoura M, Naylor RN, et al. Diabetes Presentation in Infancy: High Risk of Diabetic Ketoacidosis. Diabetes care. 2017;40(10):e147-e8.

8. Wolfsdorf JI, Glaser N, Agus M, Fritsch M, Hanas R, Rewers A, et al. ISPAD Clinical Practice Consensus Guidelines 2018: Diabetic ketoacidosis and the hyperglycemic hyperosmolar state. Pediatric diabetes. 2018;19 Suppl 27:155-77.

9. Jaeckel E, Manns M, Von Herrath M. Viruses and diabetes. Annals of the New York Academy of Sciences. 2002; 958:7-25.

10. Roivainen M, Rasilainen S, Ylipaasto P, Nissinen R, Ustinov J, Bouwens L, et al. Mechanisms of coxsackievirus-induced damage to human pancreatic beta-cells. The Journal of clinical endocrinology and metabolism. 2000;85(1):432-40.

11. Soliman AT, Al-Amri M, Alleethy K, Alaaraj N, Hamed N, De Sanctis V. Newly-onset type 1 diabetes mellitus precipitated by COVID-19 in an 8-month-old infant. Acta bio-medica : Atenei Parmensis. 2020; 91(3):ahead of print.

12. Suwanwongse K, Shabarek N. Newly diagnosed diabetes mellitus, DKA, and COVID-19: Causality or coincidence? A report of three cases. Journal of medical virology. 2021; 93(2):1150-3.

13. Chee YJ, Ng SJH, Yeoh E. Diabetic ketoacidosis precipitated by Covid-19 in a patient with newly diagnosed diabetes mellitus. Diabetes research and clinical practice. 2020; 164:108166.

14. Yang JK, Lin SS, Ji XJ, Guo LM. Binding of SARS coronavirus to its receptor damages islets and causes acute diabetes. Acta diabetologica. 2010;47(3):193-9.

15. Rabizadeh S, Hajmiri M, Rajab A, Emadi Kouchak H, Nakhjavani M. Severe diabetic ketoacidosis and coronavirus disease 2019 (COVID-19) infection in a teenage patient with newly diagnosed diabetes. Journal of pediatric endocrinology \& metabolism : JPEM. 2020;33(9):1241-3.

Figure(s) caption(s):

Figure 1. Unifocal ground-glass opacity in the apical segment of right upper lobe (RUL) of the patient

Table 1. Laboratory Data of the Patient

\begin{tabular}{lll}
\hline Investigations & Results & Reference Range \\
\hline Blood glucose & 550 & $70-100$ \\
Sodium (mEq/lit) & 158 & $135-145$ \\
Potassium (mEq/lit) & 5 & $3.5-5.5$ \\
Urea (mg/dL) & 146 & $10-36$ \\
Creatinine (gr/dL) & 0.9 & $0.2-0.4$ \\
AST (units/L) & 148 & $13-35$ \\
ALT (units/L) & 275 & $13-45$ \\
INR & 1 & $0.86-1.2$ \\
PT (seconds) & 12.5 & $11.5-15.3$ \\
PTT (seconds) & 25 & $35.1-46.3$ \\
Albumin (gr/dL) & 3.3 & $3.6-5.6$ \\
phosphorus (mg/dL) & 6.5 & $4-6.5$ \\
Calcium (mg/dL) & 4.8 & $4.4-5.9$ \\
CRP (mg/dL) & 76.5 & $0-5$ \\
ESR (mm/h) & 14 & $0-10$ \\
C-Peptide (ng/cc) & 0.7 & $0.9-4.2$
\end{tabular}




\begin{tabular}{lll}
\hline Investigations & Results & Reference Range \\
\hline WBC $\left(\times 10^{3} / \mathrm{uL}\right)$ & 4.9 & $4-10$ \\
$\mathrm{RBC}\left(\times 10^{6} / \mathrm{uL}\right)$ & 3.4 & $4.5-6.3$ \\
$\mathrm{Hb}(\mathrm{gr} / \mathrm{dL})$ & 7.7 & $12.6(11.1)$ \\
$\mathrm{Hct}(\%)$ & 22 & $36(\mathrm{~min}: 31)$ \\
Platelet $\left(\times 10^{9} / \mathrm{uL}\right)$ & 75 & $150-450$ \\
Absolute neutrophil count $\left(\times 10^{3} / \mathrm{uL}\right)$ & 3.724 & - \\
Lymphocyte $\left(\times 10^{3} / \mathrm{uL}\right)$ & 1.078 & - \\
Urine ketone & +++ severe & negative \\
Blood culture & Neg & - \\
Urine culture & Neg & - \\
PCR assays for SARS-COV-2 & positive & - \\
Anti-Glutamic Acid Decarboxylase Ab IU/ml & $<0.1$ & $<10$ \\
Anti-islet cell Ab titer & Negative & $<1 / 10$ \\
Anti-Insulin Ab & 0.1 & $<2.4$ \\
Venous blood gas $($ VBG $)$ & & \\
PH & 7.01 & $7.35-7.45$ \\
Bicarbonate $(\mathrm{mmol} / \mathrm{L})$ & 4.5 & $22-28$ \\
pCO2 $(\mathrm{mmHg})$ & 19.1 & $35-45$ \\
Base Excess & -26.5 & $-2-(+2)$ \\
\hline
\end{tabular}

AST: Aspartate Aminotransferase, ALT: Alanine aminotransferase, INR: International normalized ratio, PT: Prothrombin time, PTT: Partial thromboplastin time, CRP: C-reactive protein, ESR: Erythrocyte sedimentation rate, WBC: White blood cells, RBC: Red blood cells, Hb: Hemoglobin, Hct: Hematocrit, PCR: Polymerase chain reaction, SARS-COV-2: severe acute respiratory syndrome coronavirus 2, Ab: antibody.

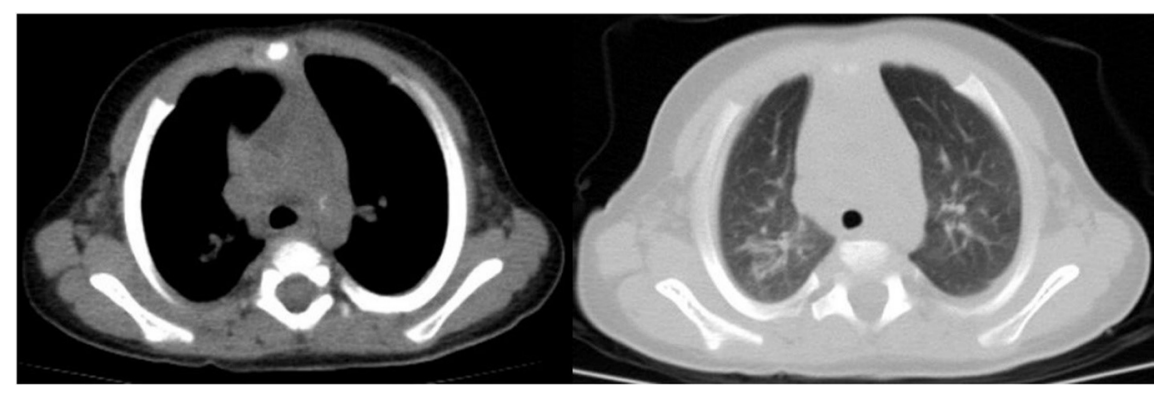

